\title{
Alterações anatomopatológicas renais em indivíduos com a sindrome da imunodeficiência adquirida
}

\author{
Renal anatomopathologic alterations in patients with acquired \\ immunodeficiency syndrome \\ Victor Alberto Laguna-Torres, Marlene Antônia dos Reis, Renato Augusto \\ Menegaz, Giovana Aund Pelá e Aluízio Prota
}

\begin{abstract}
Resumo As alterações anatomopatológicas renais foram estudadas em 119 casos de indivíduos com a síndrome da imunodeficiência humana adquirida (SIDA) no Hospital Escola da Faculdade de Medicina do Triângulo Mineiro, Uberaba MG. A partir das amostras de rim fixadas em formol, foram confeccionadas lâminas e analisadas ao microscópio de luz. Dos 119 casos estudados, 67 tiveram diagnóstico de nefrite túbulo intersticial (NTI), sendo 18 inespecíficas, 2 xantogranulomatosas e encontrou-se agente infeccioso em 48: fungos em 28 (16 Cryptococcus sp, 9 Histoplasma sp, 1 Candida $\mathrm{sp}$ e 2 Paracoccidioides brasiliensis); bactérias em 18 (9 Mycobacterium sp); vírus em 6, Citomegalovírus. Em 43 havia necrose tubular aguda (NTA). Outros diagnósticos foram: nefrocalcinose $(15,1 \%)$ e hialinose arteriolar (22,7\%). Encontrou-se também 2 casos com glomeruloesclerose segmentar focal (GESF) e um caso de hiperplasia mesangial difusa. Houve predomínio da NTI, que pode ser devido às infecções oportunistas, predominando as fúngicas; a toxicidade por drogas ou ainda devido a possível ação direta do próprio vírus. A necrose tubular aguda (NTA), foi a segunda causa em freqüência, de acometimento renal da amostra. Concluiu-se que o envolvimento renal nos pacientes com SIDA apresenta um espectro variado de processos patológicos, principalmente relacionados com infecções oportunistas, o tratamento e os procedimentos para diagnósticos, e ainda as nefropatias associadas ao vírus da imunodeficiência humana (VIH).
\end{abstract}

Palavras-chaves: Nefropatia - HIV. AIDS. Necropsia. Imunossupressão.

Abstract Renal anatomopathological lesions were studied among 119 AIDS patients from Faculdade de Medicina do Triângulo Mineiro's University Hospital (Uberaba, MG, Brazil). From formalin-fixed blocks, slides were obtained and studied by light microscopy. Of 119 patients, 67 presented tubulointersticial nefritis (TIN), 18 inespecific, 2 xantogranulomatous and infections agents were found in 48 as follows: mycosis in 28 (16 Cryptococcus sp; 9 Histoplasma sp, 1 Candida $\mathrm{sp}$ e 2 Paracoccidioides brasiliensis), bacteria in 18 (9 Mycobacterium sp), virus in 6 (Citomegalovirus). Acute tubular necrosis was found in 43 cases (36.1\%). Other diagnosis were: nefrocalcinosis (15.1\%), arteriolar hyalinosis (22.7\%), two cases of focal segmental glomerulosclerosis (1.7\%) and one case of diffuse mesangial hyperplasia (0.8\%). We conclude that the renal involvement in patients with AIDS, presents a wide spectrum of pathologies, secondary to complications related to oportunistic infections, therapeutic and diagnostic management, and the nefropathies associated to HIV.

Key-words: Nefropathy - HIV. AIDS. Autopsy. Immunossupression.

Disciplinas de Doenças Infecciosas e Parasitárias e de Patologia Geral da Faculdade de Medicina do Triângulo Mineiro (FMTM), Uberaba, MG

Endereço para correspondência: Prof Aluizio Prata. Disciplina de Doenças Infecciosas e Parasitárias, Faculdade de Medicina do Triângulo Mineiro. Rua Frei Paulino 30, 38025-180 Uberaba, MG

Fax: (034) 318-5279

E-mail: alaguna@oge.sld.pe

Recebido para publicação em 03/10/97. 
Logo após a descrição dos primeiros casos de pacientes com a síndrome da imunodeficiência adquirida (SIDA), foram identificados os comprometimentos pulmonar, cutâneo e neurológico, e diferentes agentes oportunistas foram descritos como responsáveis pelos quadros clínicos característicos 16 22. Alguns anos depois identificou-se o comprometimento renal específico, que foi caracterizado histologicamente como glomeruloesclerose segmentar e focal (GESF)5 19 20. Têm sido descritas lesões renais, tais como diferentes glomerulopatias, nefrites túbulo-intersticiais (NTI), com ou sem agentes infecciosos, dentre outras5 710111518242526 . Virtualmente, qualquer das infeções oportunistas podem acometer o parênquima renal, como conseqüência de sua disseminação. O grau de reação intersticial e o dano tubular são variados e dependem de fatores diversos como a capacidade da resposta imunológica5 25.

Grande número de portadores de SIDA vêm sendo estudados na Faculdade de Medicina do Triângulo Mineiro, em Uberaba12, inclusive sob o ponto de vista anatomopatológico. Uberaba, onde foi feito o presente trabalho, encontra-se no $44^{\circ}$ lugar de todas as cidades brasileiras em relação ao número de casos de SIDA, no período de 1980 e fevereiro de 1997, estando em 4 lugar em Minas Gerais, depois da capital do Estado e das cidades de Juiz de Fora e Uberlândia?2. Julgamos que a publicação deste material poderia trazer algum auxílio para melhor conhecimento das alterações renais nessa síndrome em nosso ambiente.

\section{MATERIAL E MÉTODOS}

O estudo foi realizado no Hospital Escola da Faculdade de Medicina do Triângulo Mineiro (HE/FMTM), Uberaba, Minas Gerais. Os doentes incluídos nesta amostra foram atendidos entre 1989 e fevereiro de 1997. Foi examinado o parênquima renal de 119 pacientes, sendo que 99 amostras provieram de necropsia e 10 foram obtidas por punção renal percutânea pós-morte com agulha de Franklin-Silverman.

De cada necropsia, obtiveram-se dois fragmentos, um de cada rim. Estes fragmentos foram fixados em formol a $10 \%$. Nas amostras colhidas por punção os fragmentos foram fixados em metacarn por quatro horas. Depois deste período, foram desidratados em concentrações crescentes de álcool, diafanizados em xilol e incluídos em parafina quente. Após microtomia dos blocos, foram obtidas dez lâminas com cortes seriados de $3 \mu \mathrm{m}$ de espessura. As lâminas foram coradas pela hematoxilina eosina (HE), tricrômico de Masson, prata metenamina (PAMS), ácido periódico de Schiff (PAS), e picrosírius. Quando necessário, foram realizadas técnicas histoquímicas para identificação de agentes infecciosos: bactérias (Gram, Fite-Faraco) e fungos (Grocott, mucicarmin).

O parênquima renal foi analisado ao microscópio de luz, seguindo um protocolo com critérios estabelecidos pela literatura8 13 . No compartimento glomerular foram estudados a celularidade, a matriz mesangial, a membrana basal e a presença de agente infeccioso. $O$ diagnóstico de glomeruloesclerose segmentar focal (GESF) foi caracterizado pelo acometimento de até $50 \%$ dos glomérulos (focal), por aumento da matriz mesangial associada com colapso capilar e conseqüente solidificação de pelo menos um lóbulo glomerular. A hipercelularidade mesangial (hiperplasia) foi caracterizada pela presença de mais de três núcleos no eixo mesangial, de aspecto global e difuso, na ausência de outras lesões glomerulares 8 . No compartimento intersticial foi investigada a presença de edema, hemorragia, infiltrado inflamatório, fibrose, calcificação e agentes infecciosos. A NTI foi caracterizada pelo infiltrado inflamatório, acompanhado ou não de fibrose intersticial. Foi considerada específica quando encontrado agente infeccioso ou inespecífica em caso contrário. $O$ diagnóstico de nefrocalcinose foi caracterizado por focos de calcificação tubular e intersticial. No compartimento tubular procurou-se a existência de necrose, degeneração, hipotrofia e calcificação. A necrose tubular aguda (NTA) foi caracterizada pela presença de túbulos dilatados com focos de membrana basal "nua", com células necróticas ou em reparação. A necrose foi identificada em túbulos sem autólise, ou seja, o encontro de células eosinofílicas com núcleos picnóticos ao lado de células preservadas da mesma secção tubular. No compartimento vascular foram examinados os capilares peritubulares e as artérias de acordo com critérios prescritos em nefropatologia. Estas artérias foram classificadas em relação ao rim, como de grande (interlobar e arqueada), médio (interlobular) e pequeno (arteríolas) calibres. Avaliou-se a existência de congestão e espessamento da parede. A hialinose arteriolar foi caracterizada pelo espessamento da íntima às custas de material amorfo eosinofílico13. 


\section{RESULTADOS}

$\mathrm{Na}$ amostra estudada de 119 pacientes com SIDA, a média das idades foi de $33 \pm 10,4$ anos, variando de 18 a 67 anos. O diagnóstico anatomopatológico renal mais freqüente foi o de nefrite túbulo intersticial (NTI) em $67(56 \%)$ casos, seguido de necrose tubular aguda (NTA) em $43(36 \%)$ pacientes, associada ou não a NTI. Em 17 (14\%) casos não foram encontradas alterações histopatológicas sugestivas de lesões renais, sendo considerados normais (Tabela 1).

Tabela 1 - Diagnósticos anatomopatológicos renais em 119 necropsias de pacientes com a síndrome da imunodeficiência adquirida.

\begin{tabular}{lrr}
\hline Diagnóstico & № & $\%$ \\
\hline Nefrite túbulo intersticial & 67 & 56,0 \\
Necrose tubular aguda & 43 & 36,1 \\
Hialinose arteriolar & 27 & 22,7 \\
Nefrocalcinose & 18 & 15,1 \\
Tumores & 4 & 3,4 \\
Vasculopatia hipertensiva & 2 & 1,7 \\
Glomeruloesclerose segmentar focal & 2 & 1,7 \\
Hiperplasia mesangial & 1 & 0,8 \\
Normais & 17 & 14,0
\end{tabular}

Obs. O somatório ultrapassa $100 \%$ porque em alguns casos foram encontrados mais de um diagnóstico.

Dos 67 casos de NTI encontrados (Tabela $2)$, em $48(71,6 \%)$ casos foram identificados agentes infecciosos. Em um caso de NTI sem agente infeccioso, havia infiltrado inflamatório mononuclear intenso, difuso, associado a polimorfonucleares do tipo eosinófilos. Em dois casos encontrou-se o padrão de NTI xantogranulomatosa caracterizada por infiltrado inflamatório mononuclear, com predomínio de plasmócitos e macrófagos repletos de lipídios (células xantomatosas). Em um destes casos havia associação com bactéria não classificada. Dos agentes infecciosos, os fungos foram identificados em $28(23,5 \%)$ casos. Do total de $20(16,8 \%)$ casos de criptococose (12 meningites e 8 disseminados), em 16 deles o fungo foi identificado no rim, correspondendo a $13,4 \%$ dos casos (Figura 1). Em 9 (7,6\%) casos encontrou-se o Histoplasma sp. O Paracoccidioides brasiliensis (Figura 2) foi encontrado em 2 casos (1,7\%). Em relação aos vírus, foram encontrados $6(5,0 \%)$ casos de Citomegalovírus (CMV) (Figura 3). Entre as 18 NTI bacterianas, em $9(7,6 \%)$ casos foi identificado Mycobacterium sp (Figura 4), nos outros casos não foi possível classificar a bactéria em gênero, sendo denominada não classificada. Em $5(4,2 \%)$ oportunidades encontrou-se 2 agentes etiológicos, sendo identificado em cada caso as seguintes associações: Cryptococcus sp e Mycobacterium $s p$, Histoplasma sp e Candida sp, Cryptococcus sp e CMV, Histoplasma sp com bactéria não classificada e NTI xantogranulomatosa com bactéria não classificada.

Tabela 2 - Agentes etiológicos das nefrites túbulo intersticiais encontrados ao exame anatomopatológico em 67 indivíduos com a síndrome da imunodeficiência adquirida.

\begin{tabular}{lc}
\hline Agente etiológico & № \\
\hline Vírus & 6 \\
$\quad$ Citomegalovirus (CMV) & \\
Bactérias & 9 \\
Mycobacterium sp & 9 \\
não classificada & \\
& \\
Fungos & \\
Cryptococcus sp & 16 \\
Histoplasma sp & 9 \\
Paracoccidioides brasiliensis & 2 \\
Candida sp & 1 \\
Inespecíficas & 18 \\
Xantogranulomatosa & 2 \\
\hline
\end{tabular}

Obs. O total ultrapassa 67 porque em algumas necropsias foi feito mais de um diagnóstico. Em 5 casos encontrou-se as seguintes associações: Cryptococcus $s p$ e Mycobacterium sp, Cryptococcus sp e CMV, Histoplasma sp e Candida sp, Histoplasma sp e bactéria não classificada, e NTi xantogranulomatosa e bactéria não classificada.

Em $2(1,7 \%)$ pacientes foi diagnosticado glomeruloesclerose segmentar focal (GESF) (Figura 5), ambos em associação com NTA. A hiperplasia mesangial difusa foi encontrada em um caso $(0,8 \%)$. Em relação aos outros diagnósticos, em 18 (15,1\%) pacientes havia nefrocalcinose, sempre como diagnóstico secundário, no contexto de uma NTI. A hialinose arteriolar foi encontrada em $27(22,7 \%)$ dos 119 pacientes (Figura 6). Destes, havia $17(63,0 \%)$ com idade inferior a 40 anos e somente um $(3,7 \%)$ acima de 60 anos. Além disso, em dois casos a hialinose estava associada a outras lesões de vasculopatia hipertensiva. Em quatro casos foram diagnosticados neoplasias, sendo dois casos de fibroma na medular, um de adenoma no córtex e em outro caso havia metástases de 

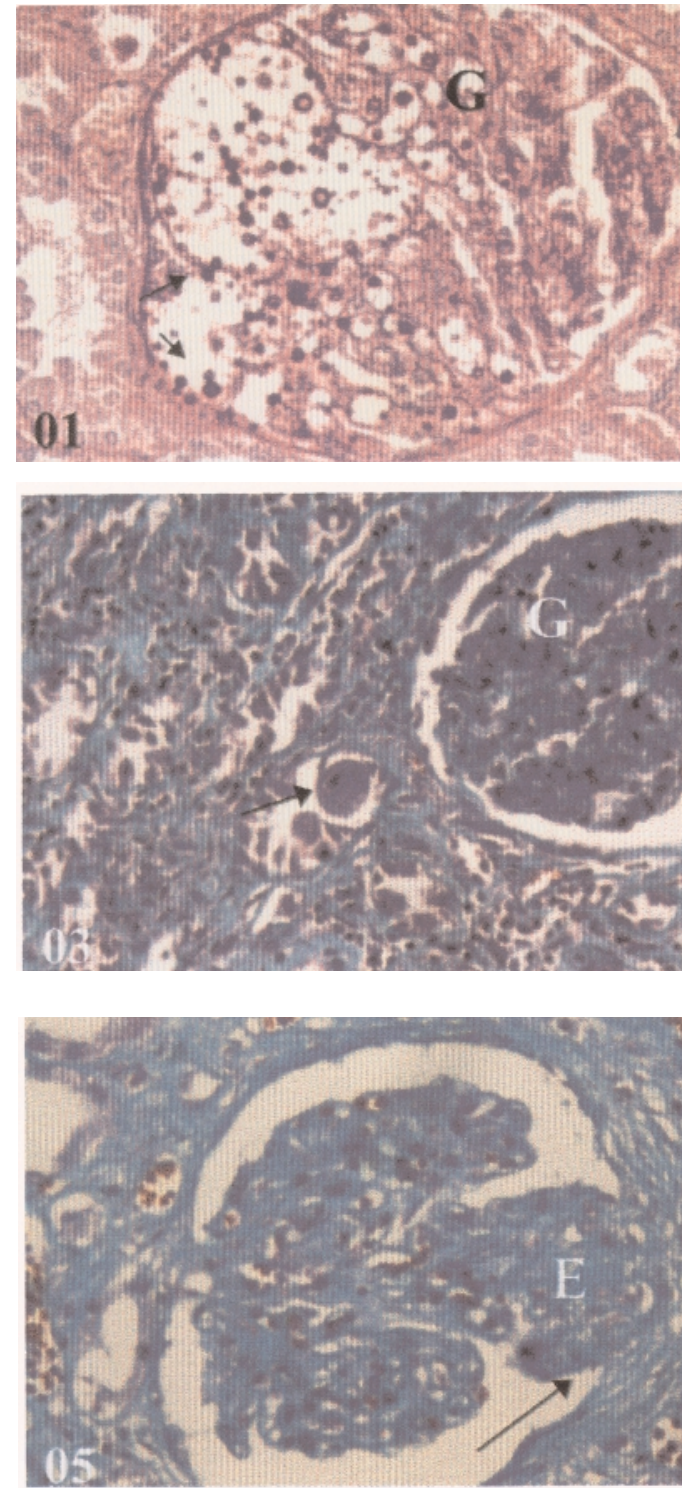
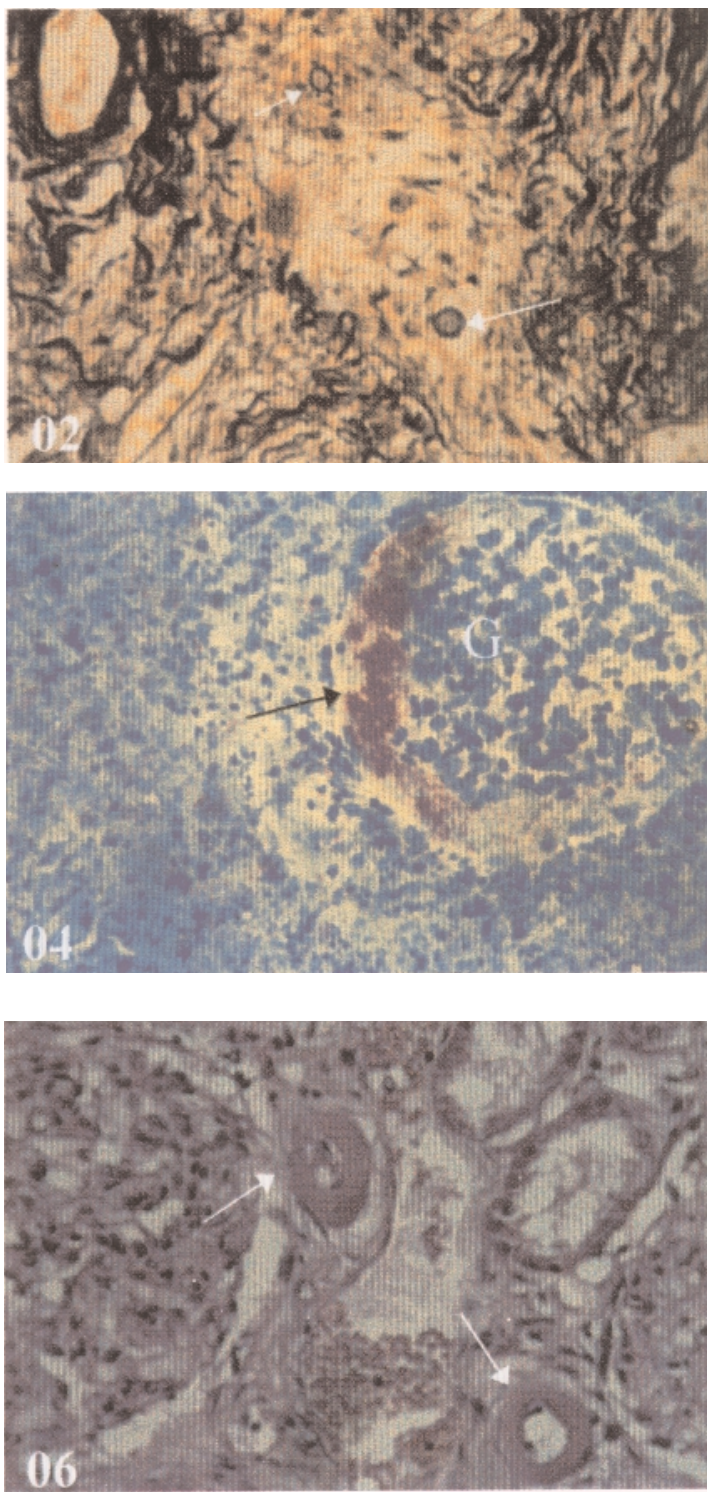

Fiqura 1 - Corte histológico de rim de indivíduo com SIDA. Nota-se glomérulo acometido por Cryptococcus neoformans. Observam-se leveduras com cápsula espessa, algumas com brotamento único (seta). (PAS, X500).

Figura 2 - Corte histológico de rim de indivíduo com SIDA. Nefrite túbulo-intersticial por Paracoccidioides brasiliensis, observase levedura com brotamento múltiplo (seta) e outra na porção inferior, com aspecto em granada (seta) (Prata metenamina, X500).

Figura 3 - Corte histológico de rim de indivíduo com SIDA. Observa-se uma célula intratubular invadida pelo Citomegalovirus (seta). O núcleo grande apresenta inclusão basofílica envolta por halo claro, a periferia do núcleo está bem marcada pela cromatina (Tricrômico de Masson, X500).

Figura 4 - Corte histológico de rim de indivíduo com SIDA. Nefrite túbulo-intersticial com intenso infiltrado inflamatório destruindo o parênquima renal. Observam-se bacilos álcool ácido resistentes (BAAR) isolados nos compartimentos intersticial, tubular e glomerular (G). Nota-se grande quantidade de BAAR no espaço de Bowman (seta). (Fite-Faraco, X500).

Figura 5 - Corte histológico de rim de indivíduo com SIDA. Glomeruloesclerose segmentar focal. Observa-se um glomérulo com aumento da matriz mesangial, caracterizando a esclerose (E) acompanhada de colapso segmentar das alças capilares, com aderência à cápsula de Bowman (seta). (Tricrômico de Masson, X500).

Figura 6 - Corte histológico de rim de indivíduo com SIDA. Hialinose arteriolar, com espessamento da parede arteriolar as custas de material amorfo eosinofílico na íntima (setas). (Hematoxilina-eosina, X500). 


\section{DISCUSSÃO}

carcinoma.

A média das idades dos 119 casos foi de $33 \pm 10,4$ anos, semelhante aos dados da literatura 15 19. Inicialmente, os autores que estudaram o rim na SIDA descreveram as lesões do compartimento glomerular, caracterizadas por GESF, chegando a encontrá-las em até $10 \%$ das amostras 19 20. No presente trabalho encontrou-se GESF em $2(1,7 \%)$ casos, prevalência semelhante aos achados de Lopes et al, no Rio de Janeiro, Brasil14, que encontraram a GESF em $6(1,1 \%)$ pacientes de 543 infectados com o vírus da imunodeficiência humana (VIH). Encontrou-se hiperplasia mesangial global e difusa em 1 caso $(0,8 \%)$. No estudo de Lopes et al14 em 40 casos de necropsias, foram descritos 3 casos $(7,5 \%)$ de hiperplasia mesangial difusa, podendo ser lesões iniciais da própria GESF. Estes mesmos autores relataram $45 \%$ dos casos com hiperplasia mesangial segmentar. No presente estudo a hiperplasia mesangial segmentar estava associada às NTI por agentes infecciosos, os quais também se encontravam no compartimento glomerular, sugerindo então que esta hiperplasia fosse secundária. A propósito, a hiperplasia mesangial segmentar não tem sido considerada como entidade isolada, pois freqüentemente é encontrada associada a outras lesões 8 . Outras modalidades de lesões glomerulares também são descritas associadas ao $\mathrm{VIH}$, como glomerulonefrite de lesões mínimas26, nefropatia $\lg \mathrm{A}^{10}$, glomerulonefrite membrano-proliferativa7 e glomerulonefrite membranosa 11.

As NTI foram as lesões mais freqüentes na presente amostra (56\%), superando os relatos da literatura, os quais variam7 1525 atingindo até $50 \% 18$. Embora as NTI tenham sido descritas em estudos iniciais de 19841920 , somente foram melhor estudadas após as pesquisas de Seney et al em 199025, seguidas pelas de Nochy et al, em 199318, estes últimos encontraram em 50\% dos 60 casos estudados, podendo estar associados ou não às glomerulopatias. As NTI podem se instalar como conseqüência de 5 grandes mecanismos que incluem infecções, drogas, nefrites mediadas por mecanismos imunológicos, as hereditárias ou metabólicas e as idiopáticas4. No paciente com SIDA, apesar da profunda imunodepressão, as lesões túbulointersticiais são freqüentes, pois, estes pacientes, estão expostos a um grande número de agentes oportunistas, responsáveis, direta ou indiretamente, pelo dano parenquimatoso. A ampla variedade dos medicamentos que estes pacientes recebem, poderiam também ser nefrotóxicos com lesões do tipo NTI4.

Dentre os agentes infecciosos, O Citomegalovirus (CMV) foi identificado em 6 $(5,0 \%)$ casos. A prevalência na literatura varia desde ausente até $13,3 \% 571525$. Dentre as bactérias, a prevalência encontrada do gênero Mycobacterium sp $(7,6 \%)$ foi maior do que a relatada pela literatura, variando de $2,5 \%$ a $4,0 \% 1525$. No entanto, outros estudos no Brasil encontraram prevalência de $25 \% 1424$. Do mesmo modo, encontrou-se maior freqüência de bactérias não classificadas $(7,6 \%)$ em relação aos $4,0 \%$ descritos por Senney25. Estas diferenças poderiam ser explicadas pelas peculiaridades geográficas.

A maioria dos agentes infecciosos encontrados no presente estudo, foram os fungos. Identificou-se o Histoplasma $s p$ em $7,6 \%$, prevalência maior do que aos $4,0 \%$ relatados na literatura25. O gênero Candida $s p$ encontrado em $0,8 \%$ foi menor do que o descrito em outros trabalhos, variando de $1,7 \%$ a $6,7 \% 515$. Embora no presente estudo tenha se identificado o Paracoccidioides brasiliensis em $1,7 \%$, não foram encontrados estudos específicos sobre o acometimento renal por este agente em paciente com SIDA na literatura. Condições climáticas e geográficas da região poderiam favorecer a proliferação de fungos como Histoplasma sp, Paraccocidioides brasiliensis ou Cryptococcus sp. A propósito, estes mesmos agentes foram encontrados em necropsias realizadas nesta região em indivíduos imunossuprimidos por transplante renal23.

O Cryptococcus sp foi encontrado em $13,7 \%$ (16 casos), freqüência maior do que os 3,1 a $8 \%$ relatados na literatura 571525 . Esses casos representam $80 \%$ dos 20 casos de criptococose diagnosticados à necropsia, mostrando que este fungo atinge o rim com facilidade. Isto já foi correlacionado com níveis muito baixos de linfócitos CD4, o que incrementa o risco de disseminação extrapulmonar27. No presente estudo existe a possibilidade de que a exposição ao Cryptococcus tenha sido maior na população estudada. Há uma relação clássica entre o Cryptococcus neoformans e o habitat dos pombos, os quais se encontram freqüentemente nas imediações do Hospital 
Escola da FMTM. O Cryptococcus neoformans, variedade gatti, tem sido isolado de ocos de Oiti, Cássia e Ficus localizados em praças públicas 29 , caracterizando a relação árvore-fungo considerada específica do Cryptococcus neoformans, variedade gatti, com o Eucalyptus camadulensis 6 . Também nas proximidades do Hospital Escola da FMTM existem eucaliptos, embora sem que se saiba qual espécie. Desta maneira, esta proximidade das árvores que poderiam albergar o fungo, de pombos que o disseminam e a imunodepressão dos pacientes criariam possíveis condições para que a população deste estudo tivessem uma freqüência maior de criptococose que as de outras séries.

A NTI, no único caso que apresentou comprometimento intersticial difuso rico em eosinófilos, poderia ter tido relação com os fármacos, tais como, cotrimoxazol, oxacilina, ceftriaxona e antiinflamatórios não-esteróides administrados ao paciente. Estas drogas podem ser nefrotóxicas 4 .

Pode-se inferir que nos pacientes em que foi identificado agente infeccioso no parênquima renal, este agente tenha sido responsável pela $\mathrm{NTI}$, ou pelo menos tenha contribuído para o processo inflamatório. Embora possa ser possível que essa presença seja circunstancial ou concomitante com o infiltrado inflamatório. Um passo importante para se determinar melhor esta situação seria identificar com técnicas adequadas o tipo celular envolvido no infiltrado 9 e até mesmo investigar a presença de antígenos do $\mathrm{VIH}$.

A NTI inespecífica pode ser produto de diferentes mecanismos. Não é possível saber com os meios utilizados, quais são os fatores que modulam a presença de infiltrados inflamatórios no parênquima renal, porém, várias evidências têm mostrado que estas células podem ser responsáveis pela presença de citocinas 11 e fatores de crescimento, tais como o fator de crescimento transformante beta (TGF-B) 1 que participam dos processos nefropáticos nos compartimentos intersticial e glomerular ${ }^{3}$. Os macrófagos são células apresentadoras de antígenos e desempenham importante papel na gênese do dano tissular, ocasionando a infiltração de linfócitos e outras células mononucleares. Esta resposta celular está alterada pela imunorregulação anormal que existe nestes pacientes 17 . A nefrocalcinose, encontrada em $15,1 \%$ freqüentemente associada a NTI, foi semelhante aos descritos na literatura, que variam de $8,4 \%$ a $38 \% 1525$.

A NTA encontrada em $43(36,1 \%)$ casos, aproxima dos $30 \%$ descritos por D'Agati et al 5 na análise de 30 casos de necropsias. No entanto, essa prevalência é maior do que a de outros estudos, $8 \%$ a $20 \% 152528$. A NTA poderia ser explicada por alterações isquêmicas ou tóxicas, tais como, depleção de volume, sepsis/choque e uso de drogas nefrotóxicas, freqüentemente observados nos pacientes com SIDA5 2128 . Numa análise de 449 indivíduos com SIDA, foram encontrados insuficiência renal aguda (IRA) em $20 \%$, sendo que o uso de drogas nefrotóxicas foi responsável por $46 \%$, e depleção de volume em $38 \% 28$. Em outra série de 146 casos de IRA/NTA, observou-se que a sepsis foi responsável direta ou indiretamente por $75 \%$ de IRA nos pacientes com SIDA 21 .

Dentre os outros processos patológicos, a hialinose arteriolar (27\%), foi pouco mais freqüente do que os $22,5 \% 15$ descritos na literatura. Esta freqüência chamou a atenção porque $60 \%$ dos pacientes acometidos tinham menos de 40 anos. Sabe-se que este processo patológico se apresenta geralmente em pacientes com diabetes mellitus, hipertensão arterial, ou em pessoas com idade avançada13. A hialinose é freqüentemente encontrada também fazendo parte da GESF8. Das neoplasias, ao contrário dos $4,2 \%$ de linfomas relatados na literatura 15 , no presente material não foi encontrado linfoma. No entanto, o adenoma cortical e metástases, encontrados em $0,8 \%$ cada, foram semelhantes aos $0,8 \%$ descritos na literatura para ambos 15 .

Concluiu-se que o envolvimento renal nos pacientes com SIDA apresenta um espectro variado de alterações anatomopatológicas, principalmente as relacionadas com as infecções oportunistas, os procedimentos terapêuticos e diagnósticos, e ainda às nefropatias associadas ao VIH.

\section{AGRADECIMENTOS}

Os autores agradecem as técnicas Valéria Ramos, Maria Aparecida da Cruz e Aparecida Martins pela confecção dos preparados histológicos.

\section{REFERÊNCIAS BIBLIOGRÁFICAS}

1. Bódi I, Abraham AA, Kimmel PL. Macrophages in human immunodeficiency virus-associated kidney diseases. American Journal of kidney diseases 24:762-767, 1994. 
2. Boletim Epidemiológico AIDS. Programa Nacional de Doenças Sexualmente Transmissíveis/AIDS. Ano IX № 5, Brasil, 1997.

3. Cattell V. Macrophages in acute glomerular inflammation. Kidney International 45:945-952, 1994.

4. Colvin R, Fang LST. Interstitial nephritis. In: Craig C; Brenner B. (ed) Renal pathology: with clinical and functional correlations. Lippincott-Raven, Philadelphia. p. 723-769, 1994.

5. D'Agati V, Cheng JI, Carbone L, Cheng JT, Cappel G. The pathology of HIV-nephropathy. A detailed morphologic and comparative study. Kidney International 35:1358-1370, 1989.

6. Ellis DH, Pfeiffer TJ. Ecology life cycle, and infectious propague of Cryptococcus neoformans. The Lancet 336:923-925, 1990

7. Gardenswartz MH, Lerner CW, Seligson GR, Zabetakis PM, Rotterdam H, Tappez ML, Michelis MF, Bruno MS. Renal disease in patients with AIDS: a clinicopathologic study. Clinical Nephrology 21:197204, 1984.

8. Jennette JC, Falk RJ. Nephritic syndrome and glomerulonephritis. In: Silva FG, D'Agati VD, Nadasdy $T$ (eds) Renal biopsy interpretation. Churchill Livingstone, New York, p. 71-114,1996.

9. Kaissling B, Le Hir M. Characterization and distribuition of interstitial cell types in the renal cortex of rats. Kidney International 45:709-720, 1994.

10. Kimmel PL, Phillips TM, Ferreira-Centeno A, FarkasSzallasi T, Abraham AA, Garret CT. Idiotypic IgA nephropathy in patients with human immunodeficiency virus infection. The New England Journal of Medicine 327:702-706, 1992.

11. Kimmel PL, Phillips TM, Ferreira-Centeno A, FarkasSzallasi T, Abraham AA, Garret CT. HIV-associated immune-mediated renal disease. Kidney International 44:1327-1340, 1993.

12. Laguna-Torres VA. Alterações renais nos pacientes com SIDA e sua expressão clínico-laboratorial e anatomopatológica. Tese de Mestrado. Universidade de Brasília, Brasília, DF, 1997.

13. Lajoie G, Silva FG. Approach to the interpretation of renal biopsy. In: Silva FG, D'Agati VD, Nadasdy T. (eds) Renal biopsy interpretation. Churchill Livingstone, New York, p. 31-70,1996.

14. Lopes GS, Marques LPG, Rioja L, Basilio-De-Oliveira CA, Oliveira AV, Nery ACF, Santos OR. Glomerular disease and human immunodeficiency virus infection in Brazil. American Journal of Nephrology 12:281287, 1992.
15. Monga G, Mazzucco G, Boldorini R, Cristina S, Giacalone A, Fortunato M, Motta M, Campobasso O. Renal changes in patients with acquired immunodeficiency syndrome: a post-mortem study on an unselected population in northwestern Italy. Modern Pathology 10:159-167, 1997.

16. Niedt GW, Schinella RA. Acquired immunodeficiency syndrome. Clinicopathologic study of 56 autopsies. Archives of Pathology and Laboratory Medicine 109:727-734, 1985.

17. Nikolic-Paterson DJ, Lan HY, Hill PA, Atkins RC. Macrophages in renal injury. Kidney International 45:S79-S82, 1994.

18. Nochy D, Glotz D, Dosquet P, Pruna A, Guettier C, Weiss L, Hinglais N, Idatte JM, Méry JP, Kazatchkine $M$, Druet $P$, Bariéty J. Renal disease associated with HIV infection: a multicentric study of 60 patients from Paris hospitals. Nephrology Dialysis Transplantation 8:11-19, 1993

19. Pardo V, Aldana M, Colton RM, Fichl MA, Jaffe D, Moskowitz L, Hensley GT, Bourgoignie JJ. Glomerular lesions in the acquired immunodeficiency syndrome. Annals of Internal Medicine 101:429-434, 1984.

20. Rao TKS, Filippone EJ, Nicastri AD, Landesman SH, Frank E, Chen CK, Friedman EA. Associated focal and segmental glomeruloesclerosis in the acquired immunodeficiency syndrome. The New England Journal of Medicine 310:669-673, 1984.

21. Rao TKS, Friedman EA. Outcome of sever acute renal failure in patients with acquired immunodeficiency syndrome. American Journal of kidney diseases 25:390-398, 1995.

22. Reichert C, O'Learly T, Levens D, Simrell C, Macher $\mathrm{ABE}$. Autopsy pathology in the acquired immune deficiency syndrome. American Journal Pathology 112:357-382, 1983.

23. Reis MA, Costa RS, Ferraz AS. Causes of death in renal transplant recipients: a study of 102 autopsies from 1968 to 1991. Journal of the Royal Society of Medicine 88:24-27, 1995.

24. Santos OR, Lopes GS. Renal complications of AIDS: a Latin American Perspective. Kidney: A Current Survey of World Literature 3:63-70, 1994.

25. Seney FD, Burns DK, Silva FG. Acquired immunodeficiency syndrome and the kidney. American Journal of Kidney Diseases 16:1-13, 1990.

26. Singer DRJ, Jenkins A, Gupta S, Evans D. Minimal change nephropathy in the immune deficiency syndrome. British Medical Journal 291:868, 1985.

27. Sorvillo F, Beall G, Turner PA, Beer VL, Kovacs AA, Kerndt PR. Incidence and factors associated with 
Revista da Sociedade Brasileira de Medicina Tropical 31:465-472, set-out, 1998.

extrapulmonary cryptococcosis among persons with HIV infection in Los Angeles country. AIDS 11:673679, 1997.

28. Valeri A, Neusy AJ. Acute and chronic renal disease in hospitalized AIDS patients. Clinical Nephrology
35:110-118, 1991.

29. Wanke B, Salmito MA, Nishikawa MM, Lázera MS. Ocos de árvores como habitat de Cryptococcus neoformans. Novos dados sobre a ecologia de ambas variedades de fungo. Revista da Sociedade 